\title{
UPOTREBA ARAPSKOG JEZIKA U GOVORU BOSANACA - DIE ARABISCHE SPRACHE IM BOSNISCHEN SPRACHGEBRAUCH
}

\section{Sažetak}

Ovaj rad traga za problematikom nastanka islamske jezičke kulture u Bosni $i$ Hercegovini $i$ istražuje upotrebu i frekventnost pojedinih arapskih riječi, odnosno fraza u govoru Bošnjaka. Istrą̌ivanje je ciljano sažeto na odredenu populaciju Bošnjaka, tj. analizirani su razgovori muslimana koji prakticiraju vjeru Islam i koji pokušavaju slijediti Poslanika, s.a.v.s., kako u kulturi ponašanja, tako i kulturi govora. Za ovu priliku je odabrana analiza jezičke upotrebe slijedecíh kur'anskohadiskih fraza: Esselamu alejkum, Alejhis-selam, Bismillah, Ma ̌̌a Allah, In ša Allah i Subhanallah.

Kur'an je prvi i glavni izvor islamske nauke. S obrirom na činjenicu da su svi muslimani obavezni upotrebljavati Allahov govor u izvornom arapskom jeriku kako bi obavljali svoje osnovne islamske du气̌nosti, ovaj rad ce sa prevodilacke perspektive poturditi cimjenicu da tekst originalnog Kur'ana nije moguce prevesti sa potpunim sadrăajem na drugi jezilk. Fraz̧e koje ce se analizirati u radu posmatraju se sa aspekta univerzalnosti. U cijelom svijetu, bez obzira koje nacije i kulture, muslimani imaju isti pozdrav - selam. Svi muslimani u svom govoru koriste fraze Bismillah, Ma ̌̌a Allah, In ̌̌a Allah i Subhanellah jer su one Božanskog porijekla.

Rezultat ovog istraživanja je prvenstveno konstatacija da su analizirane fraze dobile status jezickeih univerzalija, da nemaju potpunih bosanskih ekvivalenata $i$ da arapski jezik nije samo jezik. Arapa, nego je religijski jezik, odnosno islamski jezilk - jezik svih muslimana.

Ključne riječi: teolingvistika, arabizmi, fraze, bosanski ekvivalenti, jezičke univerzalije.

\section{„Die arabische Sprache im bosnischen Sprachgebrauch““}

In diesem Bericht wird der Beginn der islamischen Sprachkultur in Bosnien und Herzegowina und die Präsenz arabischer Wörter und Phrasen im Sprachgebrauch der Bosniaken thematisiert. Die Untersuchung beschränkt sich gezielt auf die Sprache der Moslime, die

${ }^{1}$ Islamski pedagoški fakultet u Bihaću. 
den Islam alltäglich praktizieren, deren Lebensplan und Lebensinhalt den islamischen Regeln aus dem Qur'an und der Sunna (vorbildliche Lebensweise des Propheten) unterworfen sind und die sich große Mühe geben, den Propheten Mohammed, s.a.v.s., in seiner Lebens- und Sprachkultur nachzuahmen.

Für diesen Bericht wurde der Sprachgebrauch folgender Phrasen untersucht: Esselamu alejkum, Alejhis-selam, Bismillah, Ma scha Allah, In scha Allah und Subhanellah.

Der Qur'an ist die erste und wichtigste Quelle für die islamische Lehre. Das Zitieren der originalen Verse ist eine unabdingbare Voraussetzung für die religiösen Praktiken im Islam und ebenso für das alltägliche Familienleben der Moslime. Die arabischen Phrasen, die für diese Arbeit ausgewählt wurden, werden als Sprachuniversalien definiert und gelten als unübersetzbar und unersetzbar mit bosnischen Entsprechungen. Der Gruß ist überall Selam, egal welcher Nation und Sprachgemeinschaft man angehört. Alle Moslime verwenden Phrasen wie Bismillah, Ma scha Allah, In scha Allab und Subhanellah an, weil sie göttlichen Ursprungs sind.

Am Ende der Untersuchung wird deutlich, wie einzigartig die Universalität der untersuchten Phrasen ist. Außerdem werden die Unersetzbarkeit und die Unübersetzbarkeit festgestellt und es wird konstatiert, dass die arabische Sprache nicht nur der arabischen Nation angehört, sondern sie als Sprache der Religion, bzw. Sprache der Moslime, gilt.

Stichworte: Theolinguistik, Arabismen, Phrasen, bosnische Äquivalente, Sprachuniversalien

\section{Einleitung}

In Bosnien und Herzegowina gibt es seit Jahrhunderten ein Nebeneinander verschiedener Kulturen und Religionen. Die meisten jedoch bekennen sich $\mathrm{zu}$ einer der beiden großen monotheistischen Religionsgemeinschaften, nämlich dem Christentum und dem Islam. Mehr als zwei Millionen Menschen, und das sind ungefähr 43\% der Einwohner Bosniens und Herzegowina, werden formell dem Islam zugerechnet. 
Die Verbreitung der arabischen Sprache auf den Balkangebieten geht auf die Zeit der osmanischen Herrschaft und der damaligen Islamisierung zurück. Es entwickelte sich die islamische Kultur und parallel dazu auch die islamische Sprachkultur, dessen Sprach und Verhaltensregeln aus dem heiligen Buch, dem Qur'an und den HadisSammlungen, bzw. der Sunna (der Lebenspraxis oder Tradition des Propheten Mohammed) entnommen wurden. ${ }^{2}$

Die osmanische Kultur hatte großen Einfluss auf die bosnische Literatur und den alltäglichen Sprachgebrauch der Moslime. In der heutigen bosnisch / kroatisch / serbischen Sprache ${ }^{3}$ gilt der große Anteil der sogenannten orientalistischen Wörter als der wesentliche Unterschied zwischen der serbischen oder kroatischen und der bosnischen Sprache. ${ }^{4}$

Zwar bekennen sich 43\% der Bosnier zum Islam, doch nicht jeder der sich zu einem Glauben bekennt lebt diesen Glauben und hält sich an die Glaubensregeln. Im Islam unterscheidet man zwischen den Moslimen, die sich nur formell zum Islam bekennen und den überzeugten Gläubigen, den Moslimen, die den Islam alltäglich praktizieren. Die überzeugten Moslime unterwerfen deren Lebensplan und Lebensinhalt den islamischen Regeln, so auch den islamischen Sprachregeln, die im Qur'an und der Sunne festgelegt sind. Das ist der Grund für die Präsenz der arabischen Sprache in der Sprachkultur aller Moslime, egal welcher Nationalität. Nicht nur dass die arabische Sprache eine wichtige Rolle einnimmt, sie ist ein fundamentaler Teil des Glaubens. Um dieses zu verstehen, ist es wichtig folgende aufschlussreiche Informationen über die Rolle des Qur'ans und der Hadise, bezüglich dieser Problematik, zu präsentieren.

Der Qur'an ist die erste und wichtigste Quelle für die islamische Lehre. Er ist nur in arabischer Sprache und Schrift das Original, das unverfälschte Wort Gottes. Gott sagt in Sure 43- Zuhruf, Ver 3:

2 Die beiden Quellen sind in arabischer Sprache verfasst und werden immer nur im Original zitiert. Übersetzungen gelten nicht als Gottes offenbartes Wort.

${ }^{3}$ Im heutigen Bosnien und Herzegowina sind drei Amtssprachen anerkannt: Bosnisch, Kroatisch und Serbisch. Die Unterschiede sind gering, weisen jedoch auf die nationale Identität hin

4 Škaljić, Abdulah (1973): Turcizmi u srpskohrvatskom-hrvatskosrpskom jeziku. Svjetlost, Sarajevo. 
， ，Wahrlich, Wir haben ihn zu einem Qur'an in arabischer Sprache gemacht, auf daß ihr (ihn) verstehen möget."“

Eine Übersetzung, die den Inhalt des Originaltextes nur mit menschlichen Maßstäben wiedergeben kann, wird nicht mehr als Qur'an angesehen, sondern lediglich als eine sinngemäße Übersetzung, die immer wieder Fehler und Risiken wie Fehlinterpretationen mit sich bringen kann. Übersetzungen können deshalb nicht das Zitieren der originalen Verse in den obligatorischen Gebeten, Ritualen und zu verschiedenen Anlässen ersetzen.

Hadis-Sammlungen sind die dem Propheten Mohammed zugeschriebenen Aussprüche, Mitteilungen, Anweisungen und seine

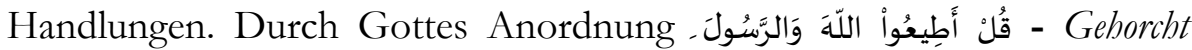
Allah und dem Gesandten (Sure 3-Imran, Vers 32) bilden die Hadise die zweite Grundlage für die islamischen Rechtlinien.

Sowohl die Verse als auch die Hadise beinhalten Verhaltensregeln nicht nur in Bezug auf religiöse Pflichten, Gebete und anderes, sondern auch auf das tägliche Leben und somit auch auf Sprachkultur der Moslime.

\section{Korpus und Zielsetzung}

Diese Arbeit ist aus der Sicht der Moslime konzipiert, die sich Mühe geben, sich an so viele Regeln, wie möglich zu halten.

Ohne Zweifel sind die orientalischen Wörter in allen Lebensbereichen zu finden. Für diese Arbeit wurde jedoch speziell nach Lexemen gesucht, die sich auf das religiöse Leben von bosnischen Moslimen beziehen. Deshalb fanden die Untersuchungen gezielt im Fastenmonat Ramadan statt, wo die meisten Moslime verschiedene religiöse Veranstaltungen organisieren, sich treffen und über religiöse Themen diskutieren. Ich nahm an den Veranstaltungen teil und verzeichnete im Laufe des Monats 245 verschiedene religiöse Begriffe. Von insgesamt 245 religiösen Begriffen waren 124, deren man arabischen Ursprung nachweisen konnte. ${ }^{5}$

${ }^{5}$ Sie wurden in arabischen Wörterbüchern „Lisanul arab“ und im arabischen originalen Korantext identifiziert. 
Im Gegensatz zu anderen Bereichen gelten die arabischen Elemente in den religiösen Begriffen, die aus dem Qur'antext stammen, als unübersetzbar und unersetzbar mit bosnischen Entsprechungen.

Das qur'anische Wort „saburi“ im Sinne von „habe Geduld“ wird nur von Moslimen benutzt, weil es im Grunde genommen noch eine wichtige Komponente beinhaltet. Saburi hat die Bedeutungen: Habe Geduld und rechne mit Allahs Belohnung. Es lohnt sich Geduld zu haben.

Im Gegensatz zu „habe Geduld“ hat das Wort „saburi“ die Funktion einer spezifischen Deutung. Allahs Wort „sabr“ wird 86-mal im Qur'an erwähnt. Das Benutzen des Wortes sabur anstatt Geduld weist auf das Spezifikum des Bosnischen in der b/k/s Sprache hin und deutet auf eine Religiosität der Person hin.

Hier muss darauf hingewiesen werden, dass nicht jedes arabische Wort den gleichen Stellenwert im Islam hat. Der Gebrauch von Sabur,

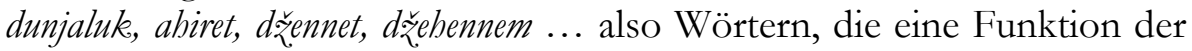
spezifischen Deutung haben, gelten nicht als eine unabdingbare Voraussetzung für die religiösen Praktiken im Islam.

Dementgegen stehen aber einzelne Lexeme und einige formelhafte Wendungen, die einen ganz besonderen religiösen Wert im Islam und somit auch der bosnisch-herzegowinischen Moslime haben. Deren Anwendung ist sozusagen durch strikte Anweisung im Qur'an und der Sunna, sie anzuwenden, Pflicht geworden.

Meine Untersuchungen werden sich nur auf die obligatorischen Phrasen beziehen. Einführend werden Wörter wie Selam und Islam näher beschrieben um die Phrasen Esselamu alejkum und Alejhis-selam besser zu verstehen.

Für diese Arbeit sind folgende Beispiele untersucht und analysiert worden:

- سلام Selam (,Friede sei mit euch“),

إسلام - Islam (Islam),

Alejhis-selam („Friede mit ihm“),

- Sallellahu alejhi ve sellem („Möge Gottes Frieden und Segen auf ihm sein"), 
- Allah (Gott)

- Bimillah (,Im Namen Gottes“),

Maschallah (,So Gottes Wille“),

Inschallah (,,So Gott will“),

Elhamdulillah (,Gott sei Dank“)

Ziel meiner Arbeit ist, die Herkunftsgeschichte, die Bedeutung und den Stellenwert dieser speziellen Wendungen aus der Sicht der bosnischen Moslime zu erläutern. Außerdem gehe ich der Frage der angeblichen Unübersetzbarkeit und Unersetzbarkeit dieser Phrasen nach, die stets auch bosnische Entsprechungen haben.

Als Grundlage für meine Präsentation ziehe ich die Textabschnitte (Verse) aus dem Heiligen Buch Qur'an und der Hadis-Sammlungen heran, die nicht nur diese besonderen Phrasen beinhalten, sondern ihre Anwendung vorschreiben. Diese vergleiche ich dann mit der bosnischen Alltagssprache.

\section{Analyse}

Einführend wird ganz kurz an die Grammatik bzw. die Wortbildung in der arabischen Sprache erinnert:

Interessanterweise ist die grammatische Norm der klassisch arabischen Sprache ursprünglich aus der einzigen Quelle, dem heiligen Qur'an entworfen, die sich auch nach $15 \mathrm{Jh}$. nicht geändert hat. ${ }^{6}$ Trotzdem ist sie, wegen der reichen Wortbildungsmöglichkeiten, der Flexibilität und der extrem konsequenten Regeleinhaltung in der Lage, neue, moderne, wissenschaftliche Begriffe, mit den bestehenden Wortwurzeln zu bilden und somit präzise Bedeutungen wiederzugeben.

Fast alle arabischen Wörter lassen sich auf drei Konsonanten, die so genannte dreiradikale Wurzel, reduzieren. Dieser Wurzel ist ein ungefährer Bedeutungsinhalt zugeordnet, die Wurzelbedeutung. Nehmen wir das bekannte Beispiel:

${ }^{6}$ Hofmann Murad, Ein philosophischer Weg zum Islam, Köln, 1983. 
K-T-B Basisbedeutung - alles, was mit Schreiben verbunden ist

Kنتاب KiTaB etwas Geschriebenes - das Buch

maKTaB ein Ort an dem geschrieben wird: das Büro, der Tisch, Arbeitsplatz

Durch die Flexionsregeln (der Wurzelerweiterung durch Hinzufügen von Vokalen oder bestimmten Konsonanten bzw. Affixen) wird der ungefähre Inhalt konkretisiert. So kann man aus einer Wurzelform um die $100 \mathrm{Wörter} \mathrm{bilden.}$

Um die Bedeutung eines schon erweiterten Wortes herauszubekommen, ist der Grundgedanke der Wurzel ausschlaggebend und die Bedeutung der Affixe leitet uns auf den konkreten Inhalt hin.

Die arabischen Wörterbücher sind logischerweise dann auch nach Wortwurzeln geordnet. Ohne das Wissen über die fundamentale Bedeutung haben wir keine Chance ein hingesagtes Wort zu verstehen. Erst wenn man die Wortwurzel entschlüsselt, ist man in der Lage, die Funktion der einzelnen hinzugefügten Buchstaben zu erkennen, sie dementsprechend $\mathrm{zu}$ vokalisieren und erst dann die Bedeutung herauszubekommen. Die Wörter werden normalerweise gar nicht vokalisiert. Für uns Lernende dieser Sprache ist es ein faszinierender Prozess, ein schweres fantasievolles Puzzlespiel, die Arabisch sprechenden jedoch beherrschen die einzelnen Grammatikregeln und entschlüsseln mit Leichtigkeit Sätze, ganze Texte, die nur aus Konsonanten bestehen. Alle Achtung!

\section{Islam (Unterwerfung)}

Das arabische Wort Islam wird in deutschen Wörterbüchern mit: Friede, Hingabe, Unterwerfung oder Aufopferung übersetzt.

Um nach der Bedeutung in arabischen Wörterbüchern nachzuschlagen, muss man es unter سلم S-L-M suchen.

Der erste Schritt war nämlich das Reduzieren des Wortes auf die Wortwurzel, also die Konsonanten der Wortwurzel entziffern.

\footnotetext{
${ }^{7}$ Im Duden Universalwörterbuch steht: völlige Hingabe (an Allah).
} 
Im Qur'an wird fast 100 mal die Wurzel S-L-M in verschiedenen Formen erwähnt. Die drei häufigsten aus dieser Wortwurzel zusammengesetzten Wörter sind Selam, Islam, Moslem

Wie schon erwähnt, die Wurzel trägt eine Hauptbedeutung, es wird sozusagen eine Plattform gegeben.

S-L-M (سَلَمَ) hat im Grunde genommen immer etwas mit Friede zu tun. (Ruhe, Segen, Ergebenheit, Rettung, Wohlbehaltenheit) Das ist der ungefähre, der weite Bedeutungsinhalt.

S-L-A-M (سلام) durch das Hinzufügen des Buchstaben Elif nach dem zweiten Konsonanten mit der grammatischen Funktion der Dehnung, bekommt es eine konkrete Bedeutung - Friede, Frieden. (In der mündlichen Sprache wird dann automatisch das Aussprechen des Vokals E nach dem S verlangt)

I-S-L-A-M (إسلام) wird darauf jetzt noch ein Elif (also der gleiche Buchstabe, aber mit einer anderen Funktion, die in der mündlichen Sprache dann automatisch das Aussprechen des Vokals I verlangt) vor die Wurzel gesetzt, verändert es den Inhalt. Islam bedeutet Frieden machen, Ruhe finden, sich ergeben ...

M-S-L-M (مسلم) der Buchstabe M vor der Wurzel deutet auf eine Person hin. (In der mündlichen Sprache wird der Vokal U verlangt) Moslem bedeutet: Einer der Frieden macht.

$\begin{array}{ll}\text { S-L-M } & \text { Alles, was mit Frieden zu tun hat } \\ \text { SELAM } & \text { Friede, Frieden } \\ \text { ISLAM } & \text { Frieden machen } \\ \text { MOSLEM } & \text { Einer, der Frieden macht }\end{array}$

Das wäre die wörtliche Bedeutung von Islam. Wie auch in anderen Sprachen hängt die letztendliche Bedeutung vom Kontext ab.

Bezogen auf die Religion und die qur'anische Bedeutung, heißt ISLAM: Frieden machen, und zwar seinen Frieden machen mit Gott, mit sich selbst und mit anderen Menschen und der Umwelt durch bedingungslose Hingabe an Allah, die Unterwerfung unter seinen Willen und seine Regeln. 
Moslime glauben fest daran, dass Gott selbst die Begriffe erwählte, was man aus dem Qur'an entnehmen kann.

Allah sagt in Sura Ali Imran Vers 19: إِنَّ الدِيَّنَ عِندَ اللَّهِ الِِْنَلَامُ ,Siehe, die Religion bei Allah ist der Islam.“ ISLAM wird noch weitere 8-mal im Qur'an in dieser Form erwähnt. Das Wort bezeichnet kein Volk, keine Nationalität und keine Person oder seine Anhänger, sondern eine für jeden Gläubigen charakteristische Handlung, „Frieden machen durch bedingungslose Hingabe“ bzw. „Unterwerfung“. Der Islam lehrt also nichts anderes als das, was sein Name beinhaltet: die Unterwerfung unter den Willen und die Gebote Allahs, die Er über den Propheten Mohammed im heiligen Buch, dem Qur'an offenbart hat.

\section{- Selam (Frieden)}

Das Wort Selam wurde in der Analyse von Islam erwähnt. Es bedeutet Friede, Frieden, Ruhe, Gelassenheit und kommt um die 40-mal im Qur'an vor. Selam ist nur die gekürzte Form von ESSELAMU ALEJKUM. Uns allen bekannt ist es als Gruß oder Abschiedswort.

Da das Fundament unseres Glaubens der Friede mit allen und allem ist, hat Gott für uns auch das Grußwort bestimmt. Gezielt ist die Wahl nicht jedem überlassen, Moslime untereinander müssen sich also mit dem Selam begrüßen und verabschieden. Gott sagt: بَإِذَا جَاءَكَكَ الَّذِينَ

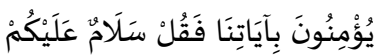

"Und wenn jene, die an Unsere Zeichen glauben, zu dir kommen, so sprich: "'Friede sei auf euch! (Sure 6-Enam, Vers 54)

Aus dem Qur'an wird ebenfalls deutlich, dass die Grußworte SELAM schon vor der Zeit der Weltschöpfung also im Jenseits, bzw. im Paradies angewendet wurden. Aus der qur'anischen Darlegung der Adam und Eva Geschichte im Paradies belegen (begründen) Moslime ihre Behauptung. Allah sagt: Selam Adam!

In den Qur'anerzählungen, die über die frühen Moslime und Propheten berichten, wird ersichtlich, dass Selam das Grußwort der vorherigen Gesandten war. Zum Beispiel schildert Allah über den Propheten Abraham und sagt:

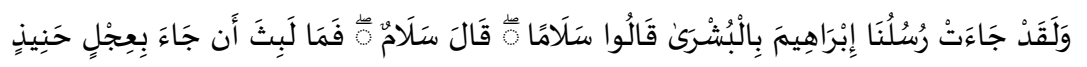


"Und es kamen Unsere Gesandten mit froher Botschaft zu Abraham. Sie sprachen: "Friede!" Er sagte: "Friede!" (Sure 11-Hud; Vers 69,70)

Gott selbst begrüßt Propheten mit dem gleichen Gruß: وَسَكَاٌْ عََلى المُرْسَلِينَ Und Friede sei auf die Gesandten! (Sure 37-Safat, Vers 181)

Auch einzeln werden Propheten namentlich genannt: Adam, Abraham, Moses, Aaron u.a.

$$
\begin{aligned}
& \text { - Nouh (Sure 37-Safat, Vers 79) } \\
& \text { Abraham (Sure 37- Safat, Vers 109) }
\end{aligned}
$$

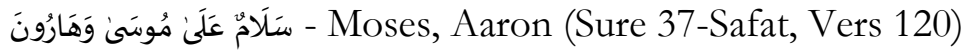

Gott verspricht außerdem, dass SELAM der Willkommensgruß im

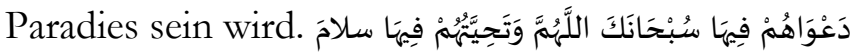

"Ihr Ruf dort wird sein: "Preis Dir, o Allah! Und ihr Gruß dort wird -Frieden sein!" (Sure 10-Junus, Vers 10)

\section{- Alejhis-selam (Friede auf ihm)}

„Alejhis-selam“, „Sallallahu alajhi we sellem“ oder „Allahumme salli ala Mohammed" (entsprechende Abkürzungen: a.s., a.s.w. und s.a.w.s.) sind verschieden Versionen der gleichen Phrase mit derselben Bedeutung „Mögen Allahs Segen und Frieden auf ihm (Mohammed) sein"

Dieser Satz oder die Phrase wird von Moslimen, nachdem man den Namen des Propheten Mohammed sagt oder hört, ausgesprochen. Dies gehört zur islamischen Sprachkultur dazu und unterstreicht den Respekt, die aufrichtige Achtung gegenüber dem Propheten a.s. verhindert jedoch die Vergötterung, seine Anbetung.

Der Qur'an befiehlt uns an mehreren Stellen, uns den Propheten

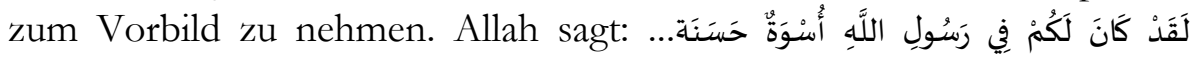
"Wahrlich, ihr habt an dem Gesandten Allahs ein schönes Vorbild ..." (Sura 33 Vers 21). Jede Nachahmung des Propheten wird als gute Tat angerechnet. Deshalb nutzen Moslime seine Aussagen und Handlungen als weitere Quelle für islamische Verhaltensregel. Sie streben danach, diese zu erlernen und sie nachzuahmen. 
Wie auch die anderen Phrasen sind diese auch aus dem Qur'an entnommen worden. Wie wir sehen können, beinhaltet diese Phrase das Wort: Selam.

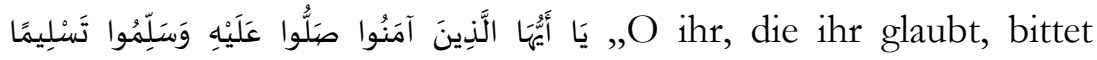
(auch) für ihn und wünscht ihm Frieden in aller Ehrerbietung." (Sure Elahzab, Vers 56)

صَلُّوا عَلَيْيِ Sallu ist ein Aufruf, eine Anweisung von Gott zum Bittgebet (die Imeperativform des Verbs beten, also: betet, bittet)

وَسَلِمِمُوا Sellimu (die Imperativform des Verbs begrüßen, im Sinne von: ihm Frieden und Segen wünschen)

Sie wird während der fünf Pflichtgebete mindestens 42-mal ausgesprochen. Außerhalb der Gebete ist es empfehlenswert bei jeder Nennung des Propheten Mohammed, ehrend „salla-llahu alejhi ve sellem“ oder kürzer „alejhis-selam“ hinzuzufügen. Im Hadis heißt es: „Wer mir den Selam wünscht, ihm wird Allah 10fach dafür seine Barmherzigkeit zeigen."

Das ist der Grund, warum bei islamischen Vorlesungen oder Vorträgen immer ein Geflüster zu hören ist, denn jedes Mal, wenn der Prophet Mohammed erwähnt wird, werden Moslime aufgefordert das Bittgebet auszusprechen und dafür verspricht Allah eine große Belohnung.

\section{- Allah (Gott)}

Redewendungen mit der Komponente Allah, in originaler Sprache, haben immer eine besondere Kraft. Aus der Vielzahl der gesammelten qur'anischen Formulierungen sind für diesen Beitrag folgende ausgewählt: Bismillah, Maschallah, Inscha Allah, Elhamdu lillah und Sallellahu alejhi ve sellem.

Das arabische Wort Allah (الله) besteht aus dem bestimmten Artikel al (ال (اله) Und dem Substantiv ilah (اله) bedeutet:jemand, der würdig ist, angebetet zu werden. Also bedeutet das Wort Allah nichts weiter als: „Der Gott“, oder „Der Eine, der es würdig ist, angebetet zu werden“ - also das, was im Bosnischen unter „Bog“ oder im Deutschen unter "Gott" verstanden wird. Es ist also gleichbedeutend, ob man Allah, Gott oder Bog sagt. Diese Auffassung wird dem Qur'an 
entnommen: "Unser Gott und euer Gott ist Einer" (Surah al-Ankebut, Vers 46). Der Glaube an Allah und somit auch der Ursprung des Wortes Allah stammt nicht, wie man vielleicht annimmt, aus der Zeit des Propheten Mohammed, denn Allah betont (genau im 111. Vers der, nach dem Propheten Josef benannten, sure) dass der Qur'an eine Bestätigung der vorherigen Offenbarungen ist ${ }^{8}$ und die Propheten Gesandte Allahs waren, die alle mit der gleichen Botschaft kamen. ${ }^{9}$ Das begründet die Präsenz des Namens Allah und der folgenden Redewendungen auch Jahrhunderte vor der Zeit des Qur'ans.

Interessanterweise haben das Wort Allah und auch die Wortverbindungen mit Allah bosnische partielle Entsprechungen, die ebenfalls präsent im alltäglichen Sprachgebrauch der Bosnier sind. Trotzdem bevorzugen viele Moslime die Verwendung von originalen Worten. Ich habe nach dem Grund und dem Unterschied gesucht.

\section{- Bismillah (Im Namen Gottes) بسم الله}

Die Phrase Bismillah mit der Bedeutung „Im Namen Gottes“ ist die gekürzte Form des Einleitungsverses im Qur’an: Bismillah- irrahmanirrahim. Die erweiterte Form beinhaltet noch zwei Attribute zu Allah: der Barmherzigste und Gnädigste.

Das vollständige Zitat setzt den ersten Vers jeder sura (Korankapitel) aus dem Qur'an fest. ${ }^{10}$ Es kommt insgesamt $116 \mathrm{Mal}$ im Qur'an vor. Dieser Satz ist der am häufigsten geschriebene und ausgesprochene arabische Satz überhaupt. In der arabischen Kalligrafie, ist er das überwiegende Motiv, in fast allen islamischen Ländern steht die Bismillah meistens als erste Phrase in Präambel, in Urkunden, Amtsbriefen und des Gleichen. Ursprünglich hat der Prophet Mohammed alle Schriftstücke (Botschaften, Einladung) mit Bismillah angefangen und ihn nehmen sich die Moslime als Beispiel.

Eine der vielen versuchten Übersetzungen würde lauten: "Im Namen des barmherzigen und gütigen Gottes“, jedoch wird dieser Satz

\footnotetext{
8 In Gestalt von Tevrat (Thora), Zebur (Psalter) und Indzil (Evangelium).

9 Der ganze Vers lautet: „Wahrlich, in ihren Geschichten ist eine Lehre für die Verständigen. Nicht ist er (Koran) eine ersonnene Geschichte, sondern eine Bestätigung dessen, was ihm vorausging, und eine Erklärung aller Dinge und eine Leitung und Barmherzigkeit für ein Volk."“

${ }^{10}$ Eine Ausnahme bildet die 9.sure, die ohne diese Einleitung anfängt.
} 
fast nie in der bosnischen Übersetzung ausgesprochen. Zum einen verliert eine Übersetzung an Inhalt und die sprachliche Bedeutung wird nur beschränkt wiedergegeben, bzw. verallgemeinert, zum anderen gibt sie nicht die Kraft und das Gefühl der vollkommenen Unterwerfung und Aufopferung ausdrücklich im Namen Allahs. Zudem sind die Anwendung und das Aussprechen der Bismilla während des Gebetes obligatorisch in arabischer Sprache. Der Satz wird mindestens 80-mal von jedem Moslem nur während der Pflichtgebete ausgesprochen. Hier sei zu erwähnen, dass ein Moslem täglich fünf Pflichtgebete verrichtet. Die Wahl der im Gebet zu rezitierenden Sura (Qur'ankapitel) wird jedem Betenden selbst überlassen und deshalb kann ein Gebet je nach Belieben ein paar Minuten oder auch viel länger dauern. Hier ist also die Rede von einer Mindestzahl von 80-mal. Da uns der Prophet Mohammed darauf hingewiesen hat, dass Gott nur die Bittgebete erhört, die mit Bismillah und Elhamdu lillah anfangen, ist es natürlich selbstverständlich, dass die Menschen jede Hinwendung zu Gott mit diesen mächtigen Sätzen anfangen und hoffen, von Gott erhört zu werden.

Außerhalb der Gebete wird der Satz vor jeder bedeutsamen Handlung oder Aufgabe und fast jeder Situation verwendet, da alle damit begonnen Taten dadurch eine für die Moslime sinnvolle Bedeutung und damit eine sich lohnende Rolle bekommen. Diese magische Phrase ausgesprochen beim Aufstehen, vor dem Frühstück, beim Anziehen, beim aus dem Haus gehen, beim Autoeinsteigen, Schreiben, Lesen, Essen, bis zum Schlafengehen, löst eine Art Garantie, Zuversicht und Trost aus. Die Formel beinhaltet das Erhoffen einer Belohnung, das Gelingen eines Vorhabens, sie ist Dank und Zeichen für Aufopferung der Taten.

Der ganze Inhalt, den wahrscheinlich nur Arabisch sprechende verstehen können, ist fast unmöglich zu übersetzen.

Deshalb bemüht sich jeder Moslem, so auch die bosnischen Moslime, vor jeder Arbeit diesen Satz im originalen arabischen Text mit seinem vollkommenen Inhalt auszusprechen, um jedes Mal mit der Aussage BISMILLAH die aufrichtige innerliche Absicht, das ganze Leben unter Allahs Willen zu stellen, zu bestätigen. 


\section{Elhamdu lillah (Gost sei Dank)}

- Elhamdulillah - Gott sei Dank, wird auch im Sinne von „Zum Glück“ oder „Gepriesen sei Gott“ gebraucht.

Die Wortverbindung besteht aus dem bestimmten Artikel El (bereits erwähnt), dem Wort Hamd mit der Wurzelbedeutung: Dank, Lob, Preis und dem Namen Allah.

Die Redewendung finden wir im ersten Vers der ersten Sure AlFatiha des Qur'ans: الحَمَدُ لله رب العالمين

Bosnische Moslime haben sowohl die arabische Phrase als auch die partiellen bosnischen Entsprechungen in ihren alltäglichen Sprachgebrauch integriert. Bevor sie sich an Gott wenden und eine Bitte aussprechen, ist es, wie bei Bismillah schon erwähnt wurde, empfehlenswert, sich vorher bei Gott im Voraus zu bedanken. Dadurch gesteht sich der Mensch letztendlich auch ein, dass er Gottes Hilfe, Segen, und Gnade bedarf. Der Prophet Mohammeds beschrieb dieses mit dem Hadis: „Die beste Art sich bei Gott zu bedanken ist mit den Worten El hamdu lillah." Als der Prophet einen Mann hörte, der seine Bitte mit Bismillah und Elhamdu lillah anfing, sagte er zu ihm: „Du hast die richtige Tür zu Gott geöffnet". Mit dem Ansatz erhofft sich der Moslem, dass seine Bitte von Gott erhört wird. Der Dank ist demnach eine Einleitung, ohne die sich eine Person nicht an Gott wenden will. Allah selbst erwähnt dieses im Qur'an und verspricht die Dankbaren zu belohnen. Er sagt in Sure Ali Imran Vers 144:Allah و سَيَجْزِي اللهُ الشَاكِرِين wird wahrlich die Dankbaren belohnen."

Das ist der Grund, warum alle Bittgebete entweder mit Bismillah oder mit Elhamdu lillah anfangen. Z.B. Die Bittgebete begleiten uns durch den ganzen Tag, sie sind nicht obligatorisch, aber da sie der Prophet zu verschiedenen Anlässen benutzte, wurden alle aufgeschrieben und den Moslimen zur Verfügung gestellt.

Die Wurzel H-M-D (ح) findet man ebenfalls in den Namen Muhammad, Mahmud, Hamid und Ahmad. Alle Namen haben die gleiche Bedeutung: ein dankbarer Untertan. 


\section{In scha Allah (Wenn es Gottes Wille ist)}

Der qur'anische Ausdruck In scha Allah besteht aus drei Lexemen. Erstens, der Konjunktion In, mit dem ein Bedingungssatz eingeleitet wird und bedeutet: wenn oder falls. Zweitens, dem Verb Scha mit der Grundbedeutung: wollen oder beabsichtigen und drittens, dem Namen Allah.

Die Phrase bedeutet übersetzt so viel wie „so Gott will oder wenn es Gottes Wille ist“. Es wird im Zusammenhang mit einem beabsichtigten oder geplanten Vorhaben benutzt. Der Moslem geht immer davon aus, dass sein ganzes Leben schon vorherbestimmt ist. Nach dem Motto: Der Mensch plant und Gott bestimmt ist der Moslem dem Gottes Willen unterworfen.

Gott selbst gibt uns die Sprachregeln vor, deshalb finden wir die Anwendung von In scha Allah direkt im Qur'an, wo es heißt:

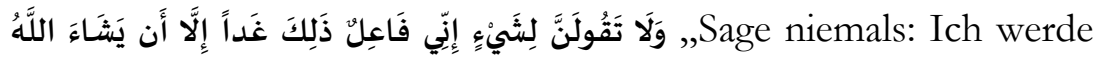
morgen etwas tun, ohne den Zusatz: In scha Allah (So Gott will)“. (Sura Kehf, Vers 23-24)

Wie wir aus diesem Vers verstehen können, wird hingewiesen, vor jedem Vorhaben oder Plan das In scha Allah hinzuzufügen.

Leider werden manchmal solche religiösen, wertvollen Ausdrücke missverstanden und gewertet und mit einer vollkommen falschen Bedeutung verwendet, was dann eine Quelle der Frustration für Moslime werden kann. In manchen Kulturen wird es nämlich im Sinne von „mal sehen“ oder „,vielleicht, vielleicht auch nicht “ benutzt und wird dann als eine Art höfliche Ablehnung oder als eine gering geschätzte Wahrscheinlichkeit interpretiert.

Die Anwendung dieser Phrase ist in Bosnien und Herzegowina sehr häufig. Auf Bosnisch übersetzt heißt es $A k o$ Bog da. Wort wörtlich übersetzt ins Deutsche wäre es Wenn Gott will. Im Gegensatz zu anderen Phrasen hat sich die bosnische Entsprechung so stark durchgesetzt, dass sie häufiger benutzt wird als das originale Arabische.

Für meine Untersuchung war jedoch der Unterschied in der Bedeutsamkeit der Aussage der arabischen Phrase und ihrer Übersetzung interessant. 
Bemerkenswert war die Anwendung der originalen Phrase in den Fällen, wo es um schwierigere Situationen geht und bewusst Gottes Hilfe gesucht wird.

Sagt die Mutter beispielsweise zum Kind: Mach dir keine Sorgen, du wirst die Prüfung In scha Allah bestehen. Die Betonung liegt im In scha Allah.

Es beinhaltet die Bitte an Gott, die Prüfung zu bestehen und die Hoffnung, es ist so vorherbestimmt.

Des Weiteren, und hier ist Pointe und der enorme Unterschied zur bosnischen Entsprechung, denn das In scha Allah verlangt, ein Nichtgelingen, als „Allahs Wille“ anzunehmen. Die Person, die diese Phrase benutzt, ist gottergeben und sieht in Gott die Allwissenheit und hat volles Vertrauen auf die Richtigkeit seiner Bestimmungen. Sie beinhaltet also das Akzeptieren und eine optimistische Haltung, falls es zum „Scheitern“ kommt.

Im Gegensatz zu der bosnischen Übersetzung $A k_{0}$ Bog $d a$, im Sinne von wenn Gott wic ist mit dem Original In scha Allah alles mit einkalkuliert, was vor allem den Zweck der Vorbeugung von negativen Folgen hat.

Die bosnische Entsprechung Ako Bog da wird als Redewendung ohne große Gedanken, geschweige denn Vorbehalte angewendet. Der Grund mag vielleicht darin liegen, dass sie von Nichtmoslimen genauso wie von Moslimen angewendet wird und sie somit für Moslime als nichtislamisch empfunden wird.

Die Redewendung Ako Bog da, mit der auf jedem Fall Gottes Wille gemeint ist, hat mit der Zeit durch die immer öftere Anwendung der originalen Phrase In scha Allah den religiösen Wert verloren. Ersetzt man zum Beispiel in einem Satz das In scha Allah mit Ako Bog da verliert es den religiösen Wert.

Im Sprachgebrauch der deutschen Moslime hat die arabische Phrase und ihre deutsche Übersetzung „So Gott will“ den gleichen religiösen Wert. 


\section{- Ma scha Allah (So wollte es Gott)}

Die Phrase Ma scha Allah bedeutet so viel wie. „Das, was Gott wollte“ oder „so war es Gottes Wille“ und weist auf die Vergangenheit hin, auf etwas, was Gott schon geschaffen hat oder so bestimmt hat.

Die arabische Phrase und die bosnische Übersetzung werden in völlig verschiedenen Kontexten benutzt. Die Bedeutung der bosnischen Übersetzung und der originalen Phrase unterscheiden sich sehr.

Wie auch in den vorherigen Phrasen finden wir den Ursprung von Ma scha Allah im Qur'an (insgesamt $5 \mathrm{Mal}$ ). Gott betont, wie Allmächtig Er ist und alles nach seinem Willen geschieht. Der Qur'an beinhaltet zum Beispiel sehr viele Geschichten mit Weisheiten, aus denen man Nutzen ziehen soll. Eine davon ist das bekannte Gleichnis der zwei Männer, einem reichen und armen Mann. Der Reiche verlor all sein Hab und Gut, weil er Gott missachtete, Ihn vergaß und undankbar wurde.

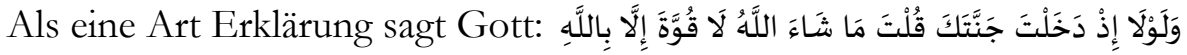

Warum hast du nicht damals, als du deinen Garten betratest, gesagt: »Das, was Allah will, (das geschieht); es gibt keine Macht außer bei Allah.« Hätte der Mann seine Verwunderung durch ein aufrichtiges Ma scha Allah ausgedrückt, wäre nichts verloren gegangen.

Die Phrase wird von Gott als eine Schutzmaßnahme gegeben. Mit diesem Ziel wird sie auch in Bosnien gebraucht.

In Bosnien wird die Phrase mit ihrem ursprünglichen Inhalt nur in arabischer Sprache, also dem Originaltext verwendet. Mit Ma scha Allah drückt man die Gottergebung und Verwunderung aus. Der Hauptgrund ist jedoch - ohne Frage - die Angst vor dem „Bösen Blick“.

Die Quelle an den Glauben und die Auffassung, gezielte Schutzsuren und Phrasen können von unheilbringendem Blick schützen, findet man im Qur'an und im Hadis. Der Prophet sagte: „Wenn jemand von euch etwas Bewundernswertes (Beeindruckendes) sieht und danach

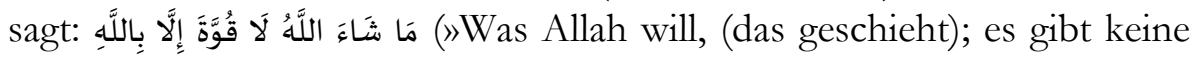
Macht außer bei Allah.«), dem kann das Auge keinen Schaden anrichten." ${ }^{\text {11 }}$

11 (Enes ibn Malik, Hadisnummer 8684) 
Die Worte Ma scha Allah sind Ausdruck der wohlmeinenden Bewunderung. Wird hingegen bei Moslimen eine Bewunderung geäußert ohne diese Worte, wird sie als Neid gewertet und löst eine Angst bei der Person, die bewundert wird, aus. Moslime reagieren dann oft und sprechen diese Phrase zum Selbstschutz aus.

Diese Redewendungen sind nicht mit Beschwörung oder Zauberei $\mathrm{zu}$ verbinden, sie haben lediglich für Moslime eine vom Unheil abwehrende, universell schützende Wirkung, die ihnen eine Art Schutz vor dem Bösen bietet.

Interessant ist jedoch die Tatsache, dass eine entsprechende Übersetzung, die wohlgemerkt im bosnischen Sprachgebrauch benutzt wird, wie „Tako je Bog odredio“- mit der Bedeutung „So hat es Gott gewollt" nur in einem völlig anderen Kontext gebraucht wird. Meistens bedient man sich dieser Redewendung um sich, oder jemanden zu trösten. (Im Todesfall, Unfall oder Ähnlichem), aber man sieht in der Übersetzung keinen Schutz.

Übrigens ist Ma scha Allah das Erste, was ein Neugeborenes zu hören bekommt. Es ist im bosnischen Kulturkreis üblich Ma scha Allah zu sagen, um Freude, Lob, Achtung auszudrücken und außerdem zu gratulieren, alles Beste dem Kind und den Eltern zu wünschen. All das und noch mehr beinhalten diese drei Wörter: Ma scha Allah. Bei jeder folgenden Lebensphase, sei es das erste Wort, der erste Schritt oder der erste Zahn, nichts wird geschildert ohne die Zugabe von Ma scha Allah. Es gehört einfach dazu.

Was noch besonders bemerkenswert bei dieser Redewendung ist, dass sie auch bei Menschen für die der Glaube eigentlich keine wichtige Rolle spielt, einen wichtigen Platz im Sprachgebrauch einnimmt.

\section{Zusammenfassung}

Zusammenfassend kann man sagen, dass wir definitiv den Ursprung und die Motivation im Qur'an und den Prophezeiungen des Propheten Mohammed, finden.

Der Grund der Unübersetzbarkeit und Unersetzbarkeit der arabischen Begriffe, oder genauer gesagt der ursprünglich qur'anischen Begriffe ist zweifellos die untrennbare Verbindung der arabischen Sprache und des Qur'ans. 
Ein wichtiger Punkt, um diese Thematik zu verstehen, ist die Erkenntnis, dass eine Übersetzung des Qur'ans nur zum besseren Verständnis dient.

SIE IST NUR EINE SINNGEMÄSSE ÜBERSETZUNG - UND KEIN QUR'AN, (sie ist nicht die wörtliche Offenbarung Gottes, deshalb kann eine noch so gute Übersetzung das Zitieren der originalen Verse in den obligatorischen Gebeten, Ritualen und den verschiedenen Anlässen nicht ersetzen.

Die konsequente Anwendung wird somit gezielt zum Mittel der Bewahrung des Originals, denn ohne Zweifel ist durch das obligatorische tägliche Rezitieren so vieler Moslime die Originalität gesichert.

Auf die Frage: Welchen Stellenwert hat die arabische Sprache bei bosnisch-herzegowinischen Moslimen, kann ich nach meinen Untersuchungen mit Gewissheit sagen: Die arabische Sprache spielt im Grunde genommen an sich keine große Rolle.

Unersetzbar sind nur die arabischen Wörter aus dem Qur'an, weil sie Gott gezielt als wesentliche Bestandteile der religiösen Praktiken und des alltäglichen Miteinanders im Leben der Moslime vorgeschrieben hat und außerdem denen die den Qur'an rezitieren und den Propheten nachahmen eine große Belohnung verspricht. ${ }^{12}$

Die Urquelle, der Qur'an ist transparent, unverändert und jedem zugänglich. Gottes originale Worte gelten als Heiligkeit und jedem Moslem Pflicht sie immer anzuwenden. Es wird keinem gestatte, irgendetwas am Original zu ändern. Das macht die arabische Sprache so einzigartig. Das Streben, Gottes Worte, die originalen unveränderten Worte, nicht die Übersetzungen von Menschen zu wiederholen.

Einzigartig ist auch die Universalität, denn die qur'anischen Wörter funktionieren wie Universalien. Der Gruß ist überall Selam, der Glaube wird von jedem mit dem Begriff Islam bezeichnet, der Vers Bismillah wird von jedem Moslem ausgesprochen. Jeder bedankt sich mit Elhamdulillah, schützt sich mit Ma scha Allah, .... Die Verse werden von

\footnotetext{
12 "Wenn jemand aus dem Buch Allahs einen Buchstaben liest, dem wird eine gute Tat zugerechnet. Für diese gute Tat werden ihm zehn Mal so viele Verdienste im Jenseits gegeben. Ich sage nicht, dass ELIF, LAM, MIM zusammen nur einen Buchstaben darstellen, sondern ELIF ist ein Buchstabe, LAM ist ein Buchstabe und MIM ist ein Buchstabe." (Sahihul buharij)
} 
allen Moslimen egal an welchem Ort und zu welcher Zeit rezitiert. Die arabische Sprache ist demnach nicht nur eine Sprache der arabischen Nation, sie ist die Sprache einer Religion. Weil diese Anordnungen von Gott selbst kommen, gibt es keine Sprachenstreitigkeiten. Das stärkt die islamische Haltung und die Gewissheit sich auf dem richtigen Weg zu befinden, um letztendlich so Allahs Zufriedenheit und seine Belohnung zu verdienen.

\section{Literaturverzeichnis}

1. Qur'an, El-Hajratu-neschr, Istanbul, 2006.

2. Ahmad, Milad Karimi (Übersetzung): Der Qur'an. Herder, Freiburg, 2009.

3. Muhammad, Asad (Übersetzung und Kommentar): Die Botschaft des Qur'ans. Patmos, Düsseldorf , 2009.

4. Buhari: El-Gamija Es-sabih, Dar Ibn Kesir, El-Jemame. Bejrut, 1987.

5. Malik: El-Muwatta. Daru Ihja Et-turas Elarabij, Kairo, 1986.

6. Ibn Menzur: Lisanul-arab. Daru Ihja Et-turas Elarabij, Bejrut, 1988.

7. Muslim: Es-sabih, Daru Ihja Et-turas Elarabij, Bejrut, 1988.

8. Kurtubi: El-Gami liabkamil Kuran, Darul Kutub El-ilmije, Bejrut, 1988.

9. Škaljić, Abdulah: Turciz̨mi u srpskobrvatskom-brvatskosrpskom jeziku. Svjetlost, Sarajevo, 1973.

10. Wagner, Andreas: Theolinguistik. Akten des 32. Linguistischen Kolloquiums in Kassel, Peter Lang, 1997.

11. Abu-r-Rida, Muhammad in Ahmad Ibn Rassoul: Die fünf Säulen des Islam. IB Verlag Islamische Bibliothek Köln, 1999.

12. Hofmann, Murad: Ein philosophischer Weg zum Islam. Köln, 1983.

13. Duden: Universalwörterbuch. Dudenverlag, Mannheim, 2003.

14. wow. islamreligion.com

15. wnw.fragenzumislam.de

16. wnw.Qur'ansuren.de 
Hatidže Burnić, MA

\title{
"DIE SPRACHE IM ARABISCHE BOSNISCHEN SPRACHGEBRAUCH"
}

\section{"THE USE OF THE ARABIC LANGUAGE IN THE SPEECH OF BOSNIAN PEOPLE"}

\begin{abstract}
This paper explores the origins of the Islamic language culture in Bosnia and Herzegovina, and the use and frequency of certain Arabic words and phrases in the speech of Bosniaks. The study was aimed at a specific population of Bosniaks, and analyzed communication between Muslims, who practice the religion of Islam, and who are trying to follow the Prophet Mubammad, in their behavior, culture and language. For this occasion, we selected analysis of linguistic usage of the following phrases from the Qur'an and Hadith: Assalamu alaikum, Alaybi salam, Bismillah, Masha Allah, Insha Allab, and Subban Allah.

The Quran is the first and main source of all Islamic sciences. Given the fact that all Muslims are obliged to use the Allah's Speech in the original Arabic language in order to perform their basic Islamic duties, this study will confirm the fact that the original text of the Qur'an can not be translated in the entire content of the second language. Phrases that will be analyzed in this paper are viewed from the aspect of universality. Muslims all over the world, regardless of their nation and culture, have the same greeting - Salam. All Muslims in their speech use the phrase Bismillah, Masha Allah, Insha Allah, and Subhan Allah because of their divine origin.
\end{abstract}

The result of this study is the statement that the analyzed phrases got the status of linguistic universals, and that they do not have equivalents in Bosnian language. Arabic language is not only the language of Arabs, but the religious language and Islamic language - the language of all Muslims.

Keywords: theolingustics, Arabism, phrases, Bosnian equivalents, linguistic universals. 


\section{م. خديجة بورنيتش}

\section{استخدام اللغة العربية في لغة البوسنويين}

\section{الخلاصة}

يتناول هذا البحث إشكالية نشوء الثقافة اللغوية الإسلامية في البوسنة والهرسك ويدرس استخدام وتكرر بعض الكلمات العربية أو العبارات في كلام البوشناق. البحث محصور بشكل هادف على أشخاص معيّنين من البشانقة، أي سيتم تحليل

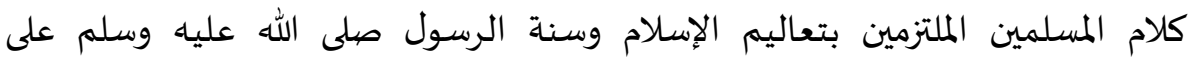
مستويين من الثقافة: ثقافة التعامل وثقافة الكلام. اخترنا لهذه المناسبة تحليل المالئل استخدام لغوي للعبارات القرآنية والحديثية التالية: السلام عليكم، عليه السلام، بسم

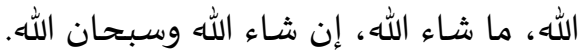

القرآن هو الأصل الأول والرئيس للعلوم الإسلامية. وبما أن أن جميع المسلمين

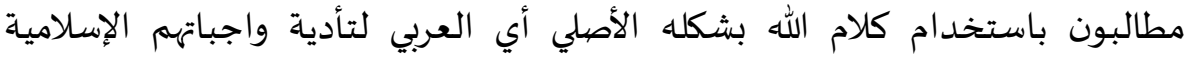

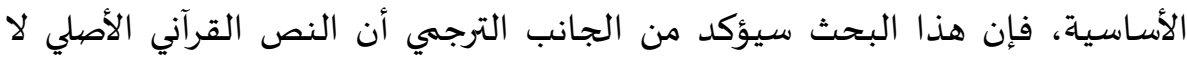

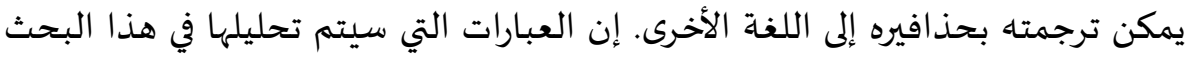

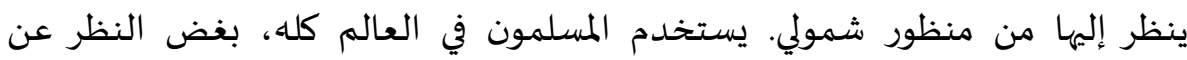

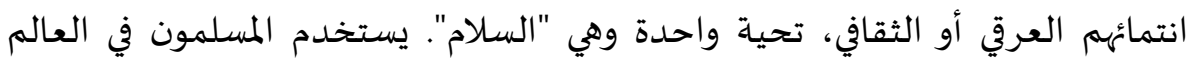

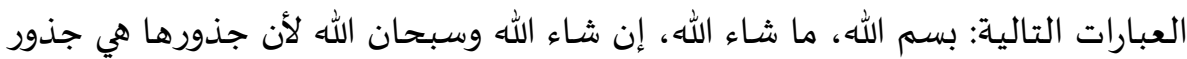
إلهية.

نتيجة هذا البحث قبل كل شيء هي التأكيد على أن العبارات المحلّلة لها وضع

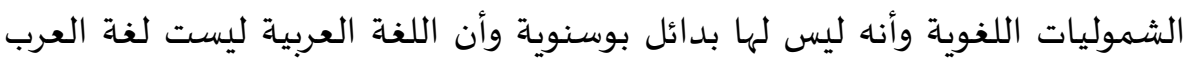
وحدهم، وإنما هي لغة الإسلام وهذا يعني أنها لغة جميع المسلمين.

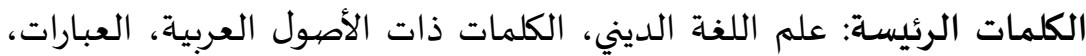

$$
\text { البدائل البوسنوية، شموليات لغوية البية البية }
$$

